\title{
EFEITO DE DIFERENTES DURAÇÕES DO ALONGAMENTO NO DESEMPENHO DE SALTOS UNIPODAIS
}

\author{
THE EFFECT OF DIFFERENT STRETCHING TIMES ON SINGLE-LEG JUMP PERFORMANCE \\ EFECTO DE DIFERENTES DURACIONES DEL ALONGAMIENTO EN EL DESEMPEÑO DE \\ SALTOS UNIPODALES
}

Paulo Henrique Marchetti ${ }^{1}, 2$

(Educador Físico)

Enrico Gori Soares

(Educador Físico)

Fernando Henrique Domingues

Oliveira Silva' (Educador Físico)

Iris Ilza de Medeiros'

(Educador Físico)

llídio dos Reis Neto ${ }^{1}$

(Educador Físico)

Charles Ricardo Lopes ${ }^{2}$

(Educador Físico)

Marco Carlos Uchida 3,4

(Educador Físico)

Reury Frank Bacurau 5

(Educador Físico)

1. Faculdade de Educação Física da Associação Cristã de Moços de Sorocaba, SP, Brasil.

2. Faculdade de Ciências

da Saúde (FACIS), UNIMEP,

Piracicaba, SP, Brasil.

3. Departamento de Ciências

Biológicas e Saúde do Centro

Universitário da Fundação

Instituto de Ensino para Osasco

(UNIFIEO), Osasco, Brasil.

4. Faculdade de Educação Física

da Universidade de Estadual de

Campinas (Unicamp),

Campinas, SP, Brasil.

5. Escola de Artes, Ciências e

Humanidades da Universidade de São Paulo (USP), SP, Brasil.

\section{Correspondência:}

Rodovia do Açúcar Km 156, Bloco 7, Sala 32, Taquaral. 13400-911.

Piracicaba, SP, Brasil.

pmarchetti@unimep.br

\begin{abstract}
RESUMO
Introdução: A prática de exercícios de alongamento é muito comum como parte do aquecimento em diversas modalidades esportivas, porém, esta prática pode ser negativa para aqueles que desejam o aprimoramento da força e potência muscular, consequentemente, o rendimento dos saltos pode ficar reduzido após exercícios de alongamento, que depende do tempo de duração e intensidade do mesmo. Objetivo: Avaliar a influência do tempo de permanência no exercício alongamento sobre o desempenho do drop jump unilateralmente (unipodal). Métodos: A amostra foi composta por 16 adultos jovens hígidos, com larga experiência em saltos. O protocolo de teste consistiu em realizar três drop jumps unipodais máximos a partir de uma plataforma de $15 \mathrm{~cm}$ sobre um tapete de salto $\left(\mathrm{Hidrofit}^{\circledR}\right)$, antes e após duas durações (i.e. uma série 3 min. de um lado, e 6 min. para outro, a escolha foi de forma aleatória) de alongamento dos músculos tríceps sural, utilizando uma intensidade entre 70-90\% da percepção subjetiva de desconforto. A análise de variância ANOVA (2x2) foi utilizada para verificar diferenças para a altura de salto, tempo de contato e flexibilidade, utilizando um nível de significância de 5\%. Resultados: Apresentaram diferenças significativas entre condições pré e pós-alongamento para as durações de 3 e 6 minutos ( $P=0,002$ e $P=0,001$, respectivamente), com a diminuição na altura máxima do salto. $O$ tempo de contato foi maior apenas para a duração de 6 minutos $(P=0,039)$. Conclusão: A realização de exercícios de alongamento do músculo tríceps sural por 3 ou 6 minutos de duração resulta na queda do desempenho do drop jump unipodal, diminuição da altura do salto vertical (para 3 e 6 min. de duração) e tempo de contato (apenas 6 ' min.), reforçando a necessidade de mais debates na área sobre exercícios de alongamento antes daqueles de potência.
\end{abstract}

Palavras-chave: exercícios de alongamento muscular, desempenho, pliometria.

\section{ABSTRACT}

Introduction: Stretching is very common as part of the warm-up in several sports modalities. However this practice can be negative to strength improvement and muscular power. Consequently, jump performance can be reduced after stretching exercises, depending on its duration and intensity. Objective: To evaluate the acute effects of different long-term stretching times on single-leg drop jump performance. Methods: The sample was composed of 16 healthy jump experienced young adult athletes. The experimental protocol consisted of performing three maximum unipodal drop jumps from a $15 \mathrm{~cm}$ high platform, and landing on a contact mat (Hidrofit ${ }^{\circledR}$ ), before and after two stretching times of the triceps surae muscles (i.e. a series of 3 minutes on one side and 6 minutes on the other, with the sides being randomly chosen), using an intensity of 70-90\% of their subjective perception of discomfort. An ANOVA (2x2) analysis was used to verify differences for the jump height, contact time and flexibility, using a significance level of $5 \%$. Results: The results showed significant differences between pre and post-stretching times of 3 and 6 minutes $(P=0.002$ and $P=0.001$, respectively). Contact time was higher only for the stretching time of 6 minutes $(P=0.039)$. Conclusion: We conclude that stretching exercises of the triceps surae muscle, for 3 or 6 minutes, resulted in a decreased performance in the single-leg drop jump, a decrease in the vertical jump height (for 3 and 6 minutes) and contact time (for 6 minutes only), reinforcing the need for more debates in this area regarding stretching before power activities.

\section{Keywords: muscle stretching exercises, performance, plyometic training.}

\section{RESUMEN}

Introducción: La práctica de ejercicios de alongamiento es muy común como parte del calentamiento en diversas modalidades deportivas. No obstante, esta práctica puede ser negativa para aquellos que desean la mejora de la fuerza y potencia muscular. Consiguientemente, el rendimiento de los saltos puede ser reducido después de ejercicios de alongamiento, que depende del tiempo de duración e intensidad del mismo. Objetivo: Evaluar la influencia del tiempo de permanencia en el ejercicio de alongamiento sobre el desempeño del drop jump unilateralmente (unipodal). Métodos: La muestra fue composta por 16 adultos jóvenes sanos, con amplia experiencia en saltos. El protocolo de test consistió en realizar tres drop jumps unipodales máximos a partir de una plataforma de $15 \mathrm{~cm}$ sobre una alfombra de salto (Hidrofit ${ }^{\circledR}$ ), antes y después de dos duraciones (i.e. una serie de 3 min. de un lado, y 6 min. para otro, la elección fue de forma aleatoria) de alongamiento de los músculos tríceps sural, utilizando una intensidad 
entre 70-90\% de la percepción subjetiva de incomodidad. El análisis de variación ANOVA (2x2) fue utilizado para verificar diferencias para la altura de salto, tiempo de contacto y flexibilidad, utilizando un nivel de significancia de 5\%. Resultados: Presentaron diferencias significativas entre condiciones prey post-alongamiento para las duraciones de 3 y 6 minutos ( $P=0,002$ y $P=0,001$, respectivamente), con la disminución en la altura máxima del salto. El tiempo de contacto fue mayor sólo para la duración de 6 minutos $(P=0,039)$. Conclusión: La realización de ejercicios de alongamiento del músculo tríceps sural por 3 ó 6 minutos de duración resulta en la caída del desempeño del drop jump unipodal, disminución de la altura del salto vertical (para 3 y 6 min. de duración) y tiempo de contacto (sólo 6 ' min.), reforzando la necesidad de más debates en el área sobre ejercicios de alongamiento antes que aquellos de potencia.

Palabras clave: ejercicios de alongamiento muscular, desempeño, pliometría.

\section{INTRODUÇÃO}

É prática comum entre atletas, técnicos ou praticantes de atividade física recreacionais utilizar o alongamento como parte do aquecimento antes de atividades de força e potência. Entretanto, as tarefas precedidas pelo alongamento podem sofrer modificações em seu desempenho e/ou coordenação em função de efeitos neurofisiológicos, hormonais, celulares e mecânicos que podem influenciar as respostas do tecido biológico em diversas atividades subsequentes como movimentos de potência e força, controle postural, afetando o desempenho físico ou a prevalência de lesões ${ }^{1-9}$. As principais modificações no desempenho físico podem estar relacionadas, principalmente as alterações no torque muscular produzido pelo alongamento, o qual modifica a relação força-comprimento influenciando os padrões de ativação neural dependendo do estresse imposto ao tecido biológico (complexo músculo-tendíneo). Tais mecanismos afetam diferentes níveis do sistema nervoso podendo influenciar no feedback proprioceptivo e consequentemente na ativação muscular ${ }^{6}$.

Os saltos possuem um caráter funcional, como na corrida', e são essenciais tanto em atividades da vida diária quanto esportivas, os quais também podem ser afetadas pelo alongamento prévio. Dentre as diferentes técnicas de salto, o drop jump é uma atividade pliométrica que envolve a queda de uma determinada altura, aterrissagem e a execução máxima do salto imediatamente após a queda. O objetivo do drop jump é melhorar a capacidade de tendões e músculos em estocarem e liberarem energia elástica quando expostos a altas forças de alongamento ${ }^{10}$. Diversos estudos mostram efeitos deletérios do alongamento para diferentes variáveis do desempenho dos saltos como altura de salto $^{1-5}$, tempo de contato ${ }^{3,5}$ e atividade eletromiográfica ${ }^{4,6}$. Isto possivelmente se deve pelo fato de que tais saltos dependem do ciclo alongamento-encurtamento, o qual utiliza recursos mecânicos teciduais (rigidez da unidade músculo-tendínea) e reflexos (fuso muscular) para o desenvolvimento de potência, e os protocolos de alongamento podem afetar diretamente tais recursos modificando o desempenho.

A relação aguda entre intensidade-duração pode afetar diretamente as estruturas da unidade músculo-tendínea de forma a alterar seu comportamento tecidual e reflexo e consequentemente afetar o desempenho esportivo subsequente. Entretanto, ainda não está claro na literatura quais são as variáveis de desempenho mais afetadas durante sessões de alongamento intensos de diferente duração. Portanto, o objetivo do presente estudo foi mensurar o efeito agudo de uma sessão de alongamento com duas diferentes durações no desempenho neuromuscular do drop jump unipodal. O objetivo do presente estudo foi mensurar o efeito agudo de uma sessão de alongamento com duas diferentes durações no desempenho neuromuscular do drop jump unipodal.

\section{MATERIAIS E MÉTODOS}

A amostra foi composta por 16 adultos jovens, hígidos, do gênero masculino (idade: $21 \pm 3$ anos; estatura: $177 \pm 8 \mathrm{~cm}$; massa: $75 \pm 13 \mathrm{~kg}$ ), alu- nos do curso de educação física e com experiência em saltos há 5 anos. Os critérios de exclusão adotados para o grupo foram os seguintes: 1) cirurgia ou lesão nos membros inferiores ou qualquer acometimento musculoesquelético em membros inferiores; 2) lesão ósteo-articular recente nos membros inferiores (menos de 1 ano); 3) terem treinado os membros inferiores nas 48 horas antecedentes ao protocolo experimental; 4) apresentar desordens neurológicas periféricas e/ou centrais. Todos os sujeitos foram informados dos procedimentos experimentais e assinaram o termo de consentimento livre e esclarecido (TCLE), aprovado pelo Comitê de Ética em Pesquisa da Universidade Metodista de Piracicaba - UNIMEP (protocolo n 73/12).

Inicialmente, os sujeitos responderam um breve questionário sobre sua dominância pedal (membro que chuta a bola) ${ }^{7}$, foram medidas a estatura e a massa e então os sujeitos realizaram uma breve sessão de familiarização com a tarefa do drop jump com ambos os membros inferiores. O protocolo de teste consistiu em realizar o drop jump unilateralmente antes e após duas durações de alongamento. O drop jump é uma técnica de salto muito difundida que envolve o ciclo alongamento-encurtamento e envolve o alongamento (ação excêntrica) e a encurtamento (ação concêntrica) ${ }^{8}$. Seu principal objetivo é melhorar a habilidade dos tendões e músculos em estocarem e liberarem energia quando expostos a altas forças de alongamento após a queda9 ${ }^{9}$.

Os saltos foram realizados em um tapete de salto da marca Hidrofit ${ }^{\circledR}$ e todos os sujeitos realizaram três drop jumps unipodais máximos antes, e após o protocolo de alongamento um drop jump unipodal máximo, sendo que cada membro inferior foi alongado com uma duração diferente (3 min ou 6 min) de forma aleatória. Durante os drop jumps todos os sujeitos foram instruídos a sair de uma plataforma de $15 \mathrm{~cm}$ de altura, cair sobre o tapete de salto e realizar o salto vertical o mais alto possível e com a maior brevidade a transição da fase excêntrica para a concêntrica. Os participantes auto selecionaram suas amplitudes articulares durante os saltos, evitando possíveis alterações no padrão de coordenação. Para minimizar possíveis influências dos membros superiores, os saltos foram realizados com os braços posicionados ao lado do corpo e as mãos apoiadas na linha da cintura. Os sujeitos foram orientados a manter seus joelhos estendidos durante toda a fase aérea e finalizar o salto com ambos os membros inferiores estendidos e com os pés sobre a área de contato do tapete de salto. Foram excluídos da análise os sujeitos que realizaram os saltos fora do tapete, não aterrissaram com o mesmo padrão de posicionamento dos pés em todos os saltos ou adotaram um posicionamento do tipo "grupado" (flexão das articulações do quadril e joelho) no momento da aterrissagem, aumentando seu tempo de voo.

Os dados analisados foram a altura de salto (AS) através do cálculo do tempo de voo dado pela fórmula: $\mathrm{AS}=\mathrm{gt}^{2} / 8$, sendo " $\mathrm{g}$ " a aceleração da gravidade $\left(9,8 \mathrm{~m} / \mathrm{s}^{2}\right)$ e "t" o tempo de voo, e o tempo de contato definido pelo tempo que o sujeito permanece no solo, entre a aterrissagem e o 
salto (transição da fase excêntrica para concêntrica) ${ }^{10}$. Somente o melhor desempenho dentre os saltos foi utilizado como parâmetro de análise.

Protocolo de Alongamento: Foram utilizadas as medidas de flexibilidade para verificar o efeito dos protocolos de alongamento. 0 protocolo consistiu em alongar passivamente os músculos responsáveis pela flexão-plantar da articulação do tornozelo (tríceps sural: gastrocnêmicos lateral e medial, e sóleo) de ambos os membros inferiores em condições de tempo de alongamento diferentes (1 série de 3 ou 6 min.). Cada membro inferior foi submetido a apenas um tipo de duração e um intervalo de 10 ' foi utilizado entre os membros. A intensidade do alongamento foi mantida pelo avaliador através da avaliação do nível de desconforto, sendo esta determinada através de uma escala subjetiva de desconforto (ESD) que varia entre 0 a 100\% [zero sem dor e 100\% o máximo desconforto imaginável durante o alongamento²]. 0 alongamento foi executado sempre pelo mesmo avaliador e a intensidade esteve entre 70-90\% da ESD. Medidas de flexibilidade (amplitude máxima de movimento passivo) foram realizadas em cada protocolo de alongamento através do movimento de dorsi-flexão, utilizando um flexímetro, nas condições de pré e imediatamente após cada protocolo de alongamento unilateral.

\section{Análise Estatística}

A normalidade e homogeneidade das variâncias foram verificadas utilizando o teste de Shapiro-Wilk e de Levene, respectivamente. Todos os dados foram reportados através da média e desvio padrão (DP) da média. A análise ANOVA (2×2) de medidas repetidas com os fatores tempo de alongamento ( 3 min e 6 min) e condição (pré e pós-alongamento) foi utilizada para verificar diferenças significativas para as seguintes variáveis: altura de salto, tempo de contato e flexibilidade. A confiabilidade das variáveis dependentes [altura de salto e tempo de contato (na condição de pré-alongamento)] foi determinada utilizando o coeficiente de correlação intraclasse (CCl). O cálculo do tamanho do feito (Effect Size, ES) foi realizado através da formula de Cohen e os resultados se basearam nos seguintes critérios: $<0.35$ efeito trivial; 0.35 0.80 pequeno efeito; 0.80-1.50 efeito moderado; e >1.5 grande efeito, para sujeitos treinados de forma recreacional baseado em Rhea ${ }^{11}$. Uma significância (a) de 5\% foi utilizada para todos os testes estatísticos, através do software SPSS versão 18.0

\section{RESULTADOS}

Foram verificadas diferenças significativas apenas entre condições pré e pós-alongamento para as duas durações (3 e 6 minutos) como mostra a tabela 1. Não foram encontradas diferenças para as condições de pré e pós-alongamento entre as diferentes durações.

o CCI foi calculado para a altura de salto e tempo de contato das condições pré-alongamento nas duas durações de alongamento (tabela 2).

Para a altura de salto foram verificadas diferenças significativas entre as condições de pré e pós-alongamento para ambas as durações: 3 minutos $(P=0,002, E S=0,84)$ e 6 minutos $(P=0,001, E S=0,56)$. Entretanto, não foram encontradas diferenças entre as condições pré-alongamento $(P=0,95)$ e pós-alongamento $(P=0,78)$ para ambas as durações do alongamento (figura 1A).

Para o tempo de contato foi verificada diferença significativa apenas entre as condições de pré e pós-alongamento na duração de 6 minutos $(P=0,039$, ES=0,71) (figura 1B).

\section{DISCUSSÃO}

Inicialmente, constatou-se que o protocolo de alongamento foi eficiente no aumento da amplitude de movimento (ADM) da articulação do tornozelo dos participantes nas condições pré e pós-alongamento, observou-se também que com o aumento da duração do alonga-
Tabela 1. Média e desvio padrão dos valores de flexibilidade antes e após diferentes durações de alongamento.

\begin{tabular}{c|c|c}
\hline \multirow{2}{*}{} & \multicolumn{2}{|c}{ Duração do Alongamento } \\
\cline { 2 - 3 } & $\mathbf{3}$ minutos & $\mathbf{6}$ minutos \\
\hline Pré-Alongamento (graus) & $17 \pm 6$ & $17 \pm 5$ \\
\hline Pós-Alongamento (graus) & $22 \pm 6$ & $23 \pm 5$ \\
\hline Valor de $P$ & 0,001 & 0,001 \\
\hline Tamanho do Efeito (ES) & 0,83 & 1,2 \\
\hline
\end{tabular}

Tabela 2. Valores do coeficiente de correlação intraclasses (CCI) e intervalo de confiança para a altura de salto e tempo de contato de ambos os membros inferiores que realizaram as diferentes durações na condição de pré-alongamento.

\begin{tabular}{c|c|c}
\hline \multirow{2}{*}{} & \multicolumn{2}{|c}{ Duração do Alongamento } \\
\cline { 2 - 3 } & $\mathbf{3}$ minutos & 6 minutos \\
\hline Altura de salto & $0,92[0,83-0,97]$ & $0,91[0,81-0,96]$ \\
\hline Tempo de contato & $0,85[0,67-0,94]$ & $0,90[0,79-0,96]$ \\
\hline
\end{tabular}
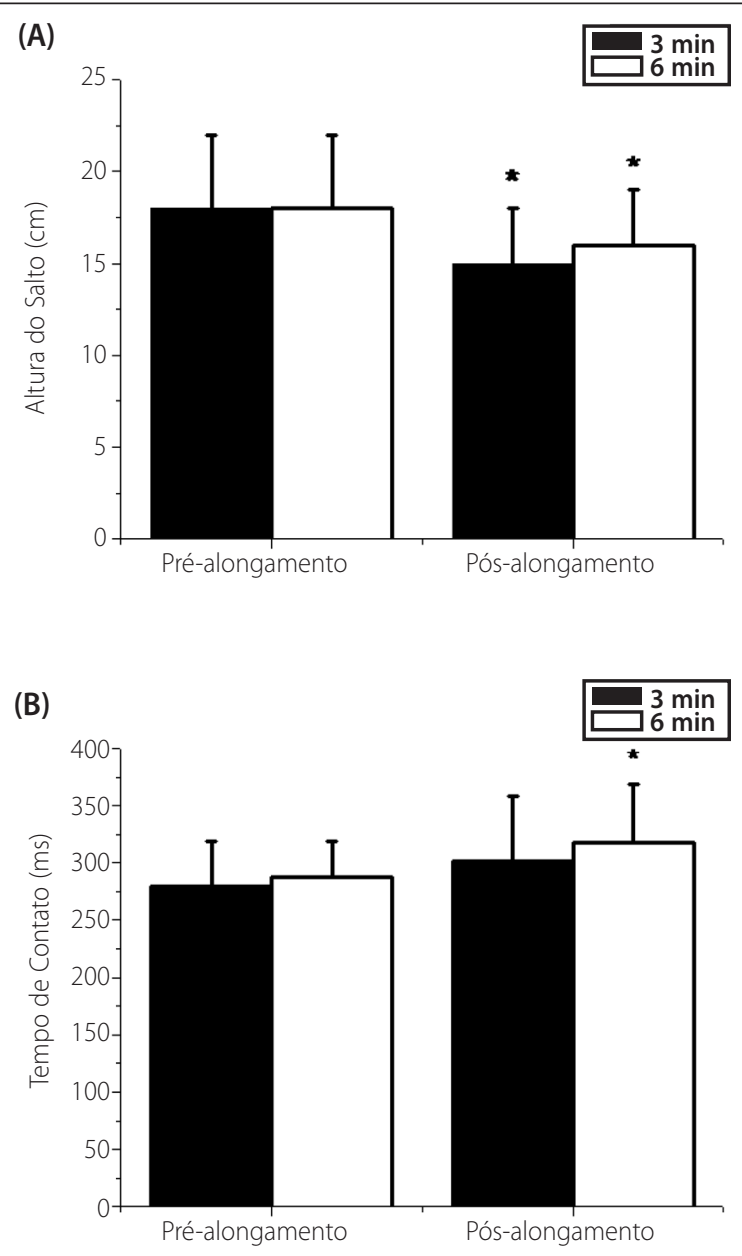

* diferença significativa entre condições de pré e pós-alongamento $(P<0,05)$.

Figura 1. Média e desvio padrão dos valores de (A) altura de salto e (B) tempo de contato para as diferentes durações do alongamento (3 e 6 minutos).

mento o tamanho do efeito foi maior. Este efeito, possivelmente, se deve ao comportamento tempo-dependente do tecido biológico que é viscoelástico, e sob estresse de alongamento apresenta menor taxa de deformação ao longo do tempo ${ }^{12-14}$. Desta forma, a duração do alongamento pode afetar o desempenho de atividades de potência e força em diferentes graus, considerando que o efeito de deformação tecidual não é linear ao longo do tempo. O estudo de Young et al. ${ }^{15}$ mostrou menores efeitos deletérios no salto em durações menores quando comparados a durações maiores $\left(1^{\prime}<2^{\prime}<4^{\prime}\right)$. Estudos mostram 
maiores quedas de desempenho quando a duração do alongamento estático é maior que 90 segundos por grupo muscular ${ }^{2}$. O presente estudo utilizou durações maiores que 90 segundos e também observou alterações negativas de desempenho em ambos os tempos de alongamento na altura de salto (para 3' e 6') e tempo de contato (para 6').

Quanto à altura de salto, observou-se queda da performance em ambos os grupos sem diferenças entre as durações do alongamento, corroborando diversos outros estudos que também mostraram queda na altura de salto após protocolos de alongamento estático ${ }^{16,17}$. A redução na altura dos saltos durante o drop jump após uma sessão aguda de alongamento passivo, pode ser atribuída às modificações mecânicas e neurofisiológicas. Quanto às alterações mecânicas decorrentes do alongamento, a redução da rigidez das estruturas periarticulares podem ter afetado a transferência das forças elásticas do ciclo alongamento-encurtamento, ocasionando diminuição do desempenho. Quanto às modificações neurofisiológicas, observam-se modificações reflexas importantes durante o alongamento, como no caso da redução dos comandos excitatórios dos aferentes "la" para os motoneurônios alfa ${ }^{5,12}$. No presente estudo, o efeito da duração do alongamento afetou negativamente a altura de salto na mesma proporção, possibilitando a influência de ambos os mecanismos (mecânico e reflexo), além de não corroborar os estudos que mostram maiores efeitos em durações mais longas de alongamento. Tais resultados também podem estar relacionados a modificações nas estratégias de salto após o alongamento, como a modificação dos torques entre articulações visando manter o desempenho.

O tempo de contato no solo (TC) é outro importante componente de desempenho dos saltos utilizando o ciclo alongamento-encurtamento, podendo variar entre 17 a 30 segundos, dependendo da técnica empregada, condição, habilidade, força e altura de queda ${ }^{9}$. O aumento do TC pode estar relacionado à maior flexão dos joelhos durante a fase de absorção de queda ou a um atraso na transmissão das forças excêntricas para concêntricas, causando a dissipação da energia elástica estocada. Durante o presente estudo foram verificadas diferenças no TC após uma única sessão de alongamento passivo apenas para a duração

\section{REFERÊNCIAS}

1. Behm DG, Button DC, Butt JC. Factors affecting force loss with prolonged stretching. Can J Appl Physiol. 2001;26:262-72.

2. Behm DG, Chaouachi A. A review of the acute effects of static and dynamic stretching on performance. Eur J Appl Physiol. 2011;111(11)2633-51.

3. Behm DG, Kibele A. Effects of differing intensities of static stretching on jump performance. Eur J Appl Physiol. 2007;101:587-94.

4. Cornwell A, Nelson AG, Sidaway B, Acute effects of stretching on the neuromechanical proprieties of the triceps surae muscle complex. Eur J Appl Physiol. 2002:86: 428-34.

5. Rubini EC, Costa AL, Gomes PS. The effects of stretching on strength performance. Sports Med. 2007;37(3): 213-24.

6. Wallmann HW, Mercer JA, McWhorter JW. Surface electromyougraphic assessment of the effects of static stretching of the gastrocnemius on vertical jump performance. J Strength Cond Res. 2005;19(3): 684-8.

7. Maulder P, J. Cronin J, Horizontal and vertical jump assessment: reliability, symmetry, discriminative and predictive ability. Phys Ther Sport. 2005;6(2):74-82.

8. Komi PV. Stretch-shortening cycle: a powerful model to study normal and fatigued muscle. J Biomech, 2000;33(10):1197-206.

9. Ball NB, Stock CG, Scurr JC. Bilateral contact ground reaction forces and contact times during plyo- do alongamento de 6 minutos. Isto, possivelmente, se deve às alterações mecânicas sofridas pela unidade músculo-tendínea, que é tempo e história dependentes, em função de suas características viscoelásti$\operatorname{cas}^{12}$ e desta forma foram mais evidentes em durações de alongamento maiores. Portanto, a maior duração do alongamento pode levar a modificações no comportamento do tecido biológico, principalmente na unidade músculo-tendínea, caracterizando um efeito de relaxamento do estresse ou deformação plástica. Tais efeitos também podem afetar a relação força-comprimento levando a modificações na contribuição dos diferentes componentes estruturais musculares, alterando a curva força-velocidade e o feedback proprioceptivo (alterando a ativação das unidades motoras) ${ }^{12}$.

Outra possível explicação para os efeitos apresentados no presente estudo é a relação intensidade-duração realizado durante o protocolo de alongamento passivo agudo. $\mathrm{O}$ aumento tanto da intensidade quanto da duração do alongamento pode afetar diretamente as características fisiológicas teciduais produzindo ruptura e desarranjos de estruturas miofibrilares (ex. titina) $)^{5}$ e/ou podem afetar o metabolismo celular devido ao efeito de isquemia e hipóxia tecidual, os quais podem levar a queda na produção de força e potência ${ }^{12}$.

O presente estudo possui algumas limitações, como a falta de verificação de possíveis modificações no padrão de salto ou atividade muscular, o efeito cruzado entre membros inferiores ou medidas de hipóxia tecidual.

\section{CONCLUSÃO}

Pode-se concluir que a realização de exercícios de alongamento do músculo tríceps sural por 3 ou 6 minutos de duração resulta na queda do desempenho do drop jump unipodal, reduzindo a da altura do salto vertical (para 3 e 6 min. de duração) e do tempo de contato (apenas para $6 \mathrm{~min}$ ).

$\overline{\text { Todos os autores declararam não haver qualquer potencial conflito de }}$ interesses referente a este artigo. metric drop jumping. J Strength Cond Res. 2010;24(10): 2762-9.

10. Marchetti PH, Uchida MC, Influence of unilateral fatigue of lower limbs on the bilateral vertical jump. Rev Bras Med Esporte. 2011;17(6): 401-4.

11. Rhea MR. Determining the magnitude of treatment effects in strength training research through the use of the effect size. J Strength Cond. Res. 2004;18(4): 918-20.

12. Avela J, Finni T, Liikavainio T, Niemelä E, Komi PV. Neural and mechanical responses of the triceps surae muscle group after 1 h of repeated fast passive stretches. J Appl Physiol. 2004;96:2325-32.

13. Taylor DC, Dalton JD Jr, Seaber AV, Garrett WE Jr. Viscoelastic properties of muscle-tendon units. The biomechanical effects of stretching. Am J Sports Med. 1990;18(3):300-9.

14. Karduna AR.Understanding the biomechanical nature of musculoskeletal tissue. J Hand Ther. 2012;25:116-22

15. Young W, Elias G, Power J. Effects of static stretching volume and intensity on plantar flexor explosive force production and range of motion. J Sports Med Phys Fitness. 2006;46:403-11.

16. Young W, Elliott J, Acute effects on static stretching proprioceptive neurmuscular facilitation stretching, and maximum voluntary contarctions on explosive force production and jumping performance. Res Q Exercise Sport. 2001;72:273-9.

17. Young WB, Behm DG. Effects of running, static stretching and practice jumps on explosive force production and jumping performance. J Sports Med. Phys Fitness. 2003;43: 21-7. 